\title{
Tiling Parity Results and the Holey Square Solution
}

\author{
Bridget Eileen Tenner \\ Department of Mathematics \\ Massachusetts Institute of Technology \\ Cambridge, MA 02139, USA \\ bridget@math.mit.edu
}

Submitted: Oct 13, 2004; Accepted: May 8, 2005; Published: May 16, 2005

Mathematics Subject Classifications: 05B45, 05C70

\begin{abstract}
We prove combinatorially that the parity of the number of domino tilings of a region is equal to the parity of the number of domino tilings of a particular subregion. Using this result we can resolve the holey square conjecture. We furthermore give combinatorial proofs of several other tiling parity results, including that the number of domino tilings of a particular family of rectangles is always odd.
\end{abstract}

\section{Introduction}

The number of domino tilings of the $2 n \times 2 n$ square with a centered hole of size $2 m \times 2 m$, a figure known as the holey square and denoted $\mathcal{H}(m, n)$, was conjectured by Edward Early to have the form $2^{\mathrm{n}-\mathrm{m}}\left(2 k_{\mathrm{m} ; \mathrm{n}}+1\right)^{2}$. Several people have worked on this problem and obtained partial results, but the general conjecture has remained open until now.

In the mid to late 1990s, Jim Propp suggested a variety of tiling enumeration questions, together with the current status of their solutions [4]. Early, a member of Propp's Tilings Research Group at MIT, posed the holey square conjecture in 1997, and Lior Pachter subsequently showed that the number of domino tilings of $\mathcal{H}(m, n)$ is $2^{\mathrm{n}-\mathrm{m}}$ times a perfect square [3]. This result follows from a factorization theorem of Mihai Ciucu [1], who was also aware of the implication but did not work specifically on Early's conjecture. In [5], Roberto Tauraso proves the result for $m=n-2$ and discusses the sequence of odd factors in this case.

Ciucu's factorization theorem, concerning the number of perfect matchings $M(G)$ of a bipartite graph $G$ with a particular symmetry property, furthermore describes the remaining squared factor as the number of domino tilings of a particular subregion of $\mathcal{H}(m, n)$. The result allows weighted edges, but for our purposes we assume that each edge has weight 1 , and simplify the statement of the theorem accordingly. Suppose that a bipartite graph $G$ is invariant under reflection across the straight line $\ell$, and the set of 
vertices of $G$ lying on $\ell$ is a cut set. The number of vertices in $\ell \cap G$ must be even, and are labeled consecutively $a_{1}, b_{1}, \ldots, a_{\mathrm{w}(\mathrm{G})}, b_{\mathrm{w}(\mathrm{G})}$. Color the bipartition classes of $G$ black and white, and remove the edges of all white $a_{\mathrm{i}}$ and black $b_{\mathrm{i}}$ vertices on one side of $\ell$, and the edges of all black $a_{\mathrm{i}}$ and white $b_{\mathrm{i}}$ vertices on the other side of $\ell$. Let $G^{+}$be the subgraph on one side of $\ell$, and $G^{-}$the subgraph on the other side of $\ell$.

Theorem 1 (Ciucu). Let $G$ be a bipartite symmetric graph separated by its axis of symmetry in the manner described above. Then

$$
M(G)=2^{\mathrm{w}(\mathrm{G})} M\left(G^{+}\right) M\left(G^{-}\right) .
$$

Writing \#R for the number of domino tilings of the region $R$, we immediately see from Theorem 1 (or rather, its dual) that $\# \mathcal{H}(m, n)=2^{\mathrm{n}-\mathrm{m}}(\# H(m, n))^{2}$ for a region $H(m, n)$ defined as follows. Consider the $2 n \times 2 n$ square, coordinatized so that the lower left corner is at the origin and the upper right corner has coordinates $(2 n, 2 n)$. Divide the square into two congruent halves by the two-unit segments

$$
\begin{aligned}
& \{[2 t, 2 t+2] \times\{2 n-(2 t+1)\}: t=0, \ldots, n-1\} \\
& \cup\{\{2 n-2 t\} \times[2 t-1,2 t+1]: t=1, \ldots, n-1\} .
\end{aligned}
$$

Now remove the center $2 m \times 2 m$ square from the region. This leaves two congruent regions, denote each by $H(m, n)$.

(a)

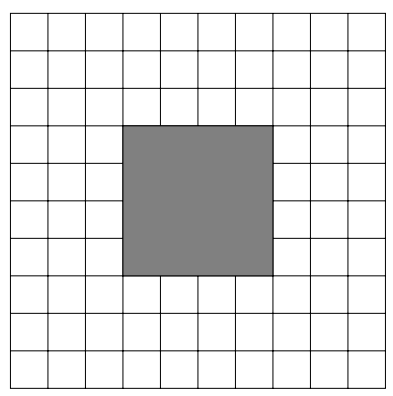

(b)

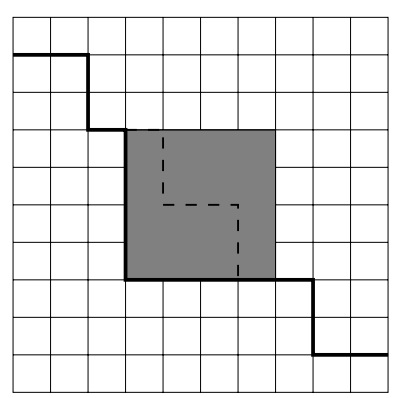

Figure 1: (a) The holey square $\mathcal{H}(2,5)$. Throughout this paper, shading indicates a portion of the figure that is excluded from the region. (b) The region below the heavy line is $H(2,5)$.

Thus the holey square conjecture reduces to determining the parity of the number of domino tilings of $H(m, n)$. This paper answers the holey square conjecture in the affirmative via a theorem about tiling parity with applications beyond the problem of the holey square. We demonstrate some of these other consequences.

As this paper solely concerns domino tilings, all tilings discussed can be assumed to be domino tilings. Following Pachter's notation in [3], we write $\#_{2} R$ for the parity of the number of tilings of $R$. A region is the dual of a finite connected induced subgraph of $\mathbb{Z}^{2}$. When only concerned with the configuration of part of a region, we may only draw this portion, indicating that the undrawn portion is arbitrary. 


\section{A tiling parity result}

This section presents a theorem regarding the parity of the number of tilings of a region. The result depends only on a local property of the region, and makes no further restrictions. Before stating this property, a few definitions are necessary.

Definition 1. A region $R$ has an $(\{s, t\} ; 1)$-corner if there is a convex corner in $R$ where the segments bounding this corner have lengths $s$ and $t$. For $p>1$ and $\min \{s, t\} \geq 2$, an $(\{s, t\} ; p)$-corner is a $(\{1, s\} ; 1)$-corner, a $(\{1, t\} ; 1)$-corner, and $p-2$ distinct $(\{1,1\} ; 1)$ corners configured as in Figure 2.

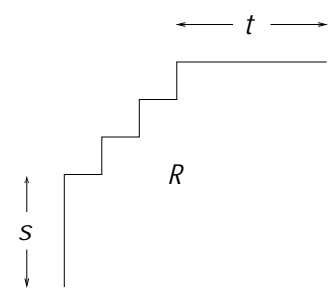

Figure 2: An $(\{s, t\} ; 4)$-corner.

Definition 2. If the segment of length $s$ in an $(\{s, t\} ; p)$-corner forms an $\left(\left\{s, t^{\prime}\right\} ; p^{\prime}\right)$-corner at its other endpoint, then each of these corners is walled at $s$.

Definition 3. An $(\{i, j\} ; p)$-strip is a subregion of $i+j+2 p-3$ squares that has an $(\{i, j\} ; p)$-corner.

The local property mentioned earlier requires that a particular subregion not have any "inconvenient holes." We use a notion of completion to describe the avoidance of such holes, and precisely define it as follows.

Definition 4. An $(\{s, t\} ; p)$-corner in a region $R$ is 2 -complete if $2 \leq \min \{s, t\}$. For $2<i \leq \min \{s, t\}$, the corner is $i$-complete if the following conditions are met:

(a) Let $C$ be the $(\{i, i\} ; p)$-strip in the $(\{s, t\} ; p)$-corner. Let $x$ and $y$ be the two squares adjacent to the ends of $C$ but not along the edges forming the $(\{s, t\} ; p)$-corner. If either $x$ or $y$ is in $R$, then the $(\{i-1, i-1\} ; p)$-strip between $x$ and $y$, inclusively, all of whose squares are adjacent to $C$, must also be a subregion of $R$.

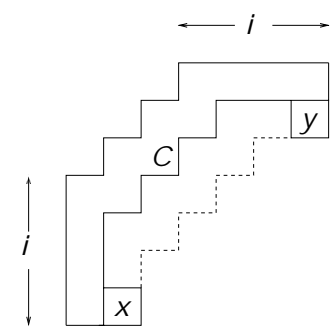


(b) Consider the $\left(\left\{s^{\prime}, t^{\prime}\right\} ; p\right)$-corner formed by removing $C$ from $R$. If $2 \leq i-2 \leq$ $\min \left\{s^{\prime}, t^{\prime}\right\}$, then this corner must be $j$-complete for $j=2, \ldots, i-2$.

In the process of determining whether or not an $(\{s, t\} ; p)$-corner is $k$-complete, the largest potential subregion of $R$ to be examined is

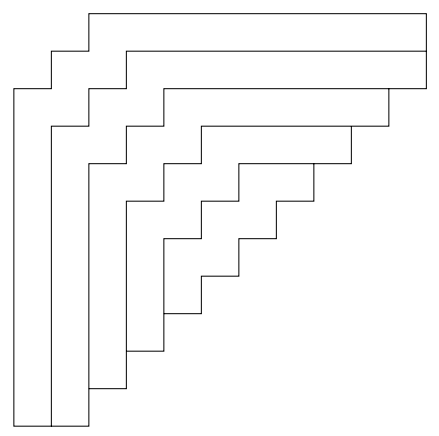

where there are $\lceil k / 2\rceil$ strips: one $(\{k, k\} ; p)$-strip, one $(\{k-1, k-1\} ; p)$-strip, one $(\{k-$ $3, k-3\} ; p)$-strip, one $(\{k-5, k-5\} ; p)$-strip, $\ldots$, concluding with a $(\{3,3\} ; p)$-strip if $k$ is even, or a $(\{2,2\} ; p)$-strip if $k$ is odd.

Definition 5. For $2 \leq k \leq \min \{s, t\}$, an $(\{s, t\} ; p)$-corner is complete up to $k$ if that corner is $i$-complete for $i=2, \ldots, k$.

For example, if $R$ has an $(\{s, t\} ; 1)$-corner that is complete up to 3 , then this corner must have one of the following forms.
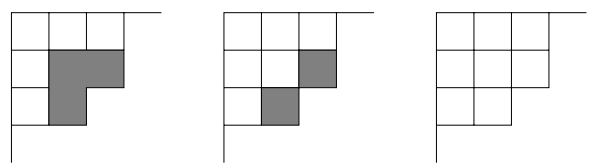

Similarly, an $(\{s, t\} ; 2)$-corner that is complete up to 3 is as follows.
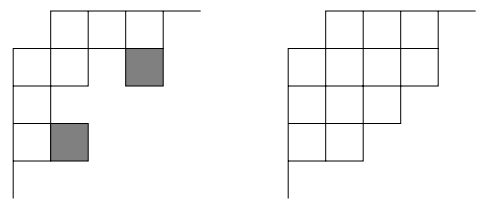

If an $(\{s, t\} ; 1)$-corner is complete up to 4 , then it must also be complete up to 3 so the above drawings together with the definition of 4-completeness require that all such corners are depicted below.
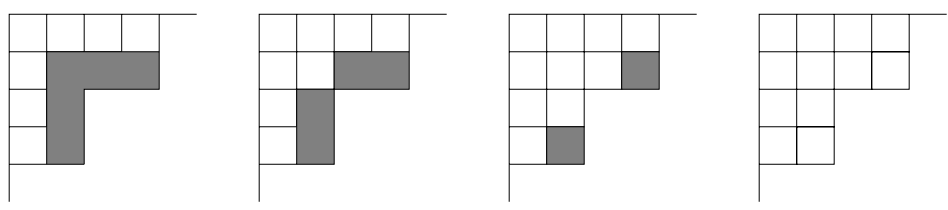
The different tilings of $R$ can be categorized by the manner in which an $(\{s, t\} ; p)$ corner is tiled. For example, if $R$ has an $(\{s, t\} ; 4)$-corner, then

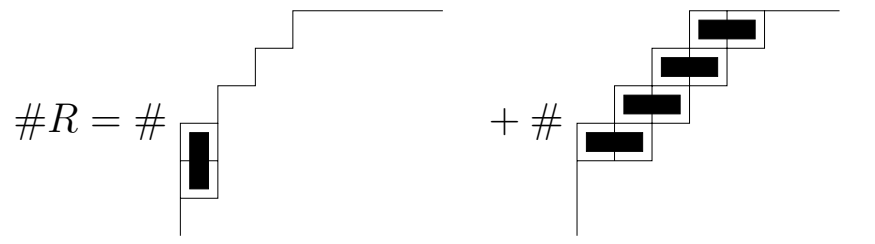

Suppose $R$ has an $(\{s, t\} ; 1)$-corner where $2 \leq \min \{s, t\}$. Then

$$
\# R=\# \mathbf{\square}+\# \begin{array}{r}
\mathbf{\square} \\
\mathbf{\square}
\end{array}
$$

where the corner drawn in each of the above figures is the particular $(\{s, t\} ; 1)$-corner in $R$. If $R$ does not include the entire region tiled in one of these figures, that term is zero. The first two of these figures tile the same subregion of $R$, so

$$
\# _ { 2 } R = \# _ { 2 } \longdiv { \square } . \# _ { 2 }
$$

Theorem 2 (Parity Theorem). Suppose that a region $R$ has an $(\{s, t\} ; p)$-corner. For any $2 \leq k \leq \min \{s, t\}$, if this corner is complete up to $k$, then

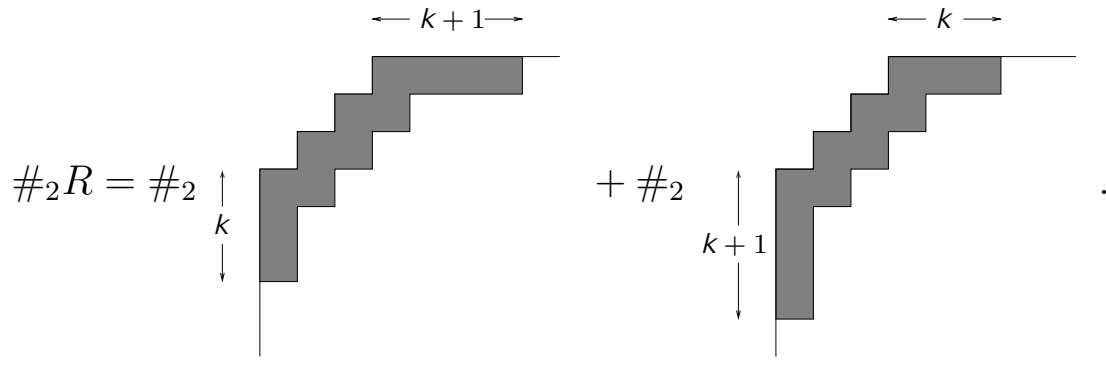

If $p=1$, then (1) also holds for $k=1$. Furthermore, for any $p$, if $s \leq t$, the corner is complete up to $s$, and the corner is walled at $s$, then

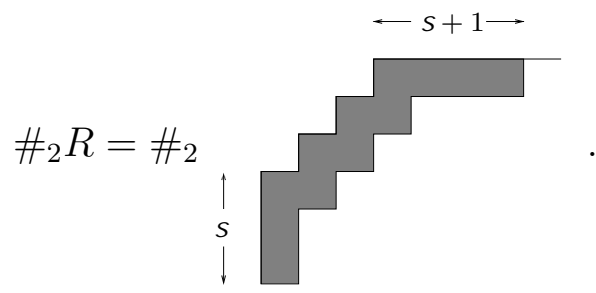

Proof. We first inductively show that for fixed $p$, the statement is true for all $(\{s, t\} ; p)$ corners complete up to $k \leq \min \{s, t\}$. We will then induct on $p$. If (1) holds for a 
particular $k$ and fixed $p$, then certainly (2) holds if there is a wall at $s=k$, since one of the tilings pictured in the statement of (1) is impossible.

Suppose that $p=1$. That (1) holds for $k=1$ is trivial, and the case $k=2$ was shown above. Assume that the theorem holds for all $k<K \leq \min \{s, t\}$, and suppose that an $(\{s, t\} ; 1)$-corner of $R$ is complete up to $K$. This corner must also be complete up to $K-1$, so apply (1) with $k=K-1$.

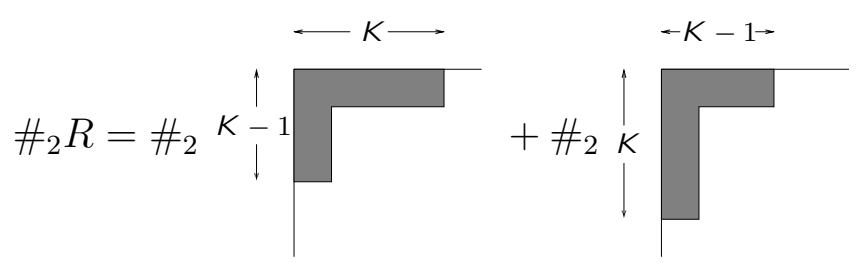

In the first of these regions, there are two ways to tile the square next to the shorter leg of the removed region.

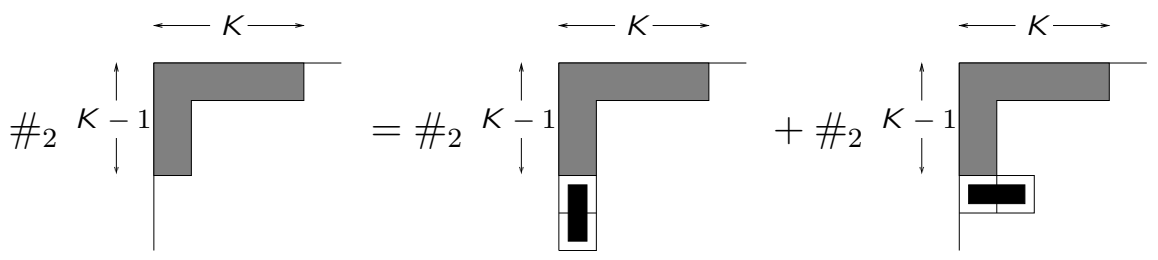

The latter of these creates a $(\{K-2, K-1\} ; 1)$-corner walled at $K-2$, and this corner is complete up to $K-2$ because of the inductive definition of completeness. Applying (2) for $k=K-2$ yields

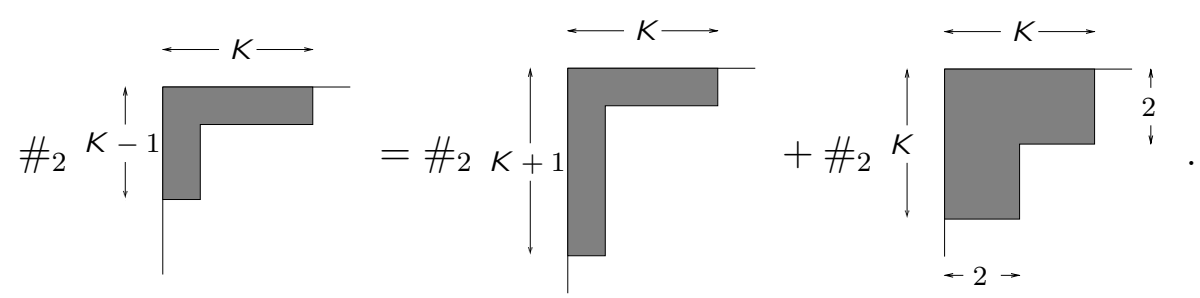

There is an analogous equation for the second region on the right-hand side of (3), and the last term in (4) also appears in this. As we are considering parity, these terms cancel, leaving

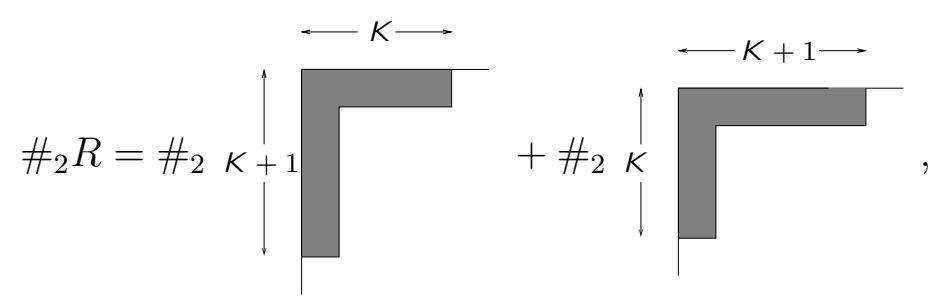

proving (1) for $k=K$, and consequently (2) for $k=K$. Thus the result holds for all $(\{s, t\} ; 1)$-corners. 
Now assume that the theorem is true for all $1 \leq p<P$, and suppose that there is an $(\{s, t\} ; P)$-corner. Consider the $(\{s, 1\} ; 1)$-corner in this $(\{s, t\} ; P)$-corner. This corner is covered by a vertical domino or a horizontal domino, giving

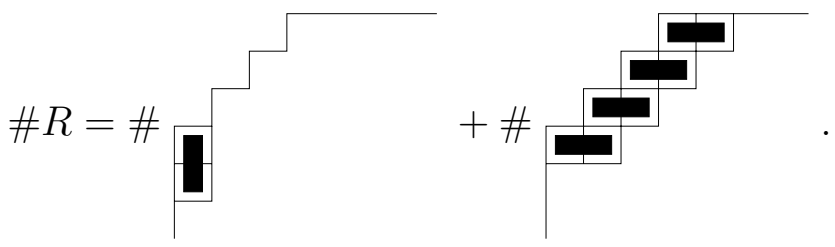

The first of these creates a $(\{3, t\} ; P-1)$-corner which is necessarily complete up to 2 , while the second creates a $(\{2, s-1\} ; 1)$-corner. To the former, apply the theorem for $p=P-1$ and $k=2$, and apply the theorem for $p=1$ and $k=1$ to the latter. Both results are already known by the induction hypothesis. Thus

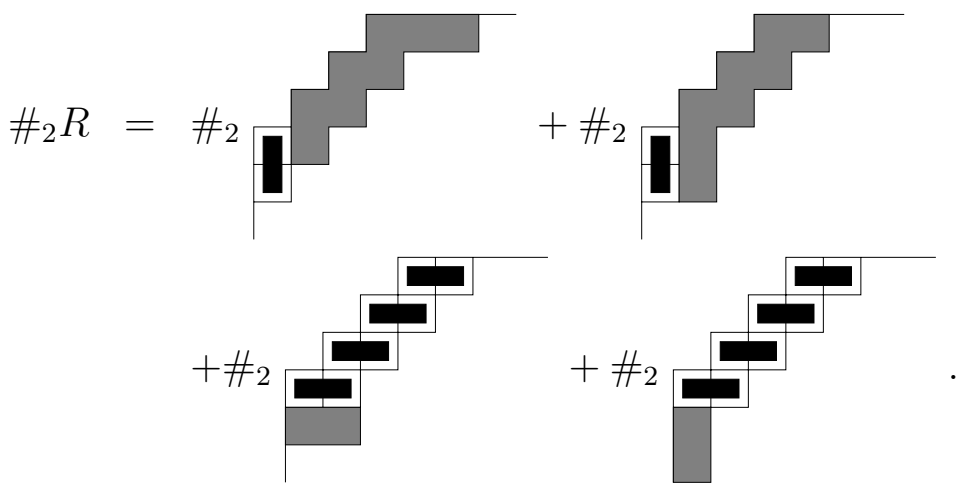

Two summands tile the same region, so they cancel and the theorem holds for $k=2$.

Now suppose that for this $P$, the theorem holds for all $2 \leq k<K \leq \min \{s, t\}$, and that there is an $(\{s, t\} ; P)$-corner of $R$ complete up to $K$. As in the case of a $(\{s, t\} ; 1)$ corner, this corner must also be complete up to $K-1$, so we can apply (1) for $k=K-1$. The remainder of the proof follows analogously to the case $p=1$.

\section{The holey square}

As discussed in the introduction, the resolution of the holey square conjecture reduces to determining the parity of the number of tilings of the region $H(m, n)$. In any tiling of $H(m, n)$, one particular domino must always be in place. In Figure 1(b), this is the domino occupying the two bottom-rightmost squares. Let $H^{\prime}(m, n)$ be $H(m, n)$ with these two squares removed, so $\# H(m, n)=\# H^{\prime}(m, n)$, and in particular these numbers have the same parity. To prove the conjecture we need to prove that $\#_{2} H(m, n)=1$.

Corollary 1. For all $m$ and $n>m, \# \mathcal{H}(m, n)=2^{\mathrm{n}-\mathrm{m}}\left(2 k_{\mathrm{m} ; \mathrm{n}}+1\right)^{2}$, where the factor $2 k_{\mathrm{m} ; \mathrm{n}}+1$ is equal to $\# H(m, n)$. 
Proof. Induct on $n-m$. The base case $n=m+1$ is trivial, as $\#_{2} H(m, m+1)=1$ and $\mathcal{H}(m, m+1)$ can be tiled in two ways. Now assume that $\#_{2} H(m, n)=1$. Consider the region $H^{\prime}(m, n+1)$ which has a $(\{2 n, 2 n+1\} ; 1)$-corner walled at $2 n$ and complete up to $2 n$. Apply the parity theorem to this corner, specifically (2). The subregion indicated by the right-hand side of (2) is actually $H(m, n)$ reflected across the line

$y=x$. Therefore $\#_{2} H^{\prime}(m, n+1)=\#_{2} H(m, n)=1$. This completes the proof since $\#_{2} H^{\prime}(m, n+1)=\#_{2} H(m, n+1)$, answering affirmatively the question posed by Early, and giving a combinatorial meaning to the odd factor in $\# \mathcal{H}(m, n)$.

Analogous to $\mathcal{H}(m, n)$, let $\mathcal{H}^{\circ}(m, n)$ be the $(2 n+1) \times(2 n+1)$ square with a centered hole of size $(2 m+1) \times(2 m+1)$.

Corollary 2. For all $m$ and $n>m$, $\# \mathcal{H}^{\circ}(m, n)=2^{\mathrm{n}-\mathrm{m}}\left(2 k_{\mathrm{m} ; \mathrm{n}}^{\prime}+1\right)^{2}$.

Proof. The proof is analogous to the proof of the previous corollary, and once again the odd factor $2 k_{\mathrm{m} ; \mathrm{n}}^{\prime}+1$ is the number of domino tilings of a particular region.

\section{Further applications of the parity theorem}

In addition to determining the number of domino tilings of the holey square, the parity theorem can be applied to other regions. One easy consequence is the following.

Corollary 3. If a region $R$ has an $(\{s, s\} ; p)$-corner that is complete up to $s$ and walled at $s$ along both sides, then $\# R$ is even.

Proof. Both of the tilings depicted in (1) are impossible, so both terms on the right side of the equation are zero.

P. W. Kasteleyn [2] gave an exact formula for the number of tilings of an $a \times b$ rectangle, denoted $N(a, b)$. When $a$ and $b$ are both even, this is:

$$
\# N(a, b)=\prod_{i=1}^{\mathrm{a}=2} \prod_{j=1}^{\mathrm{b}=2}\left(4 \cos ^{2} \frac{i \pi}{a+1}+4 \cos ^{2} \frac{j \pi}{b+1}\right) .
$$

Kasteleyn matrices provide a more general procedure for determining the number of tilings of particular regions, but, unfortunately, many consequences that can be proved using Kasteleyn matrices do not have combinatorial interpretations. Combinatorialists desire to remedy such situations, and perhaps also use combinatorial methods to obtain results that were not apparent by solely employing Kasteleyn matrices. For example, the parity of $\# N(a, b)$ is not at all obvious from (5). However, each corner of $N(a, b)$ is complete up to $\min \{a, b\}$, so we can apply the parity theorem to obtain the following result. 
Corollary 4. For all positive integers $k$ and $n, \# N(k n,(k+1) n)$ is odd.

Proof. To describe the process more precisely, suppose that $N(k n,(k+1) n)$ is oriented so that the sides of length $k n$ are vertical. Apply the parity theorem to the upper left corner to remove the $(\{k n, k n+1\} ; 1)$-strip. Similarly, if the values of $k$ and $n$ are sufficiently large, remove the $(\{n-1, n\} ; 1)$-strip from the upper right corner, the $(\{(k-1) n,(k-1) n+1\} ; 1)$-strip from the lower right corner, and the $(\{2 n-2,2 n-1\} ; 1)$-strip from the lower left corner (where each of these corners are of subsequent subregions of $N(k n,(k+1) n))$.

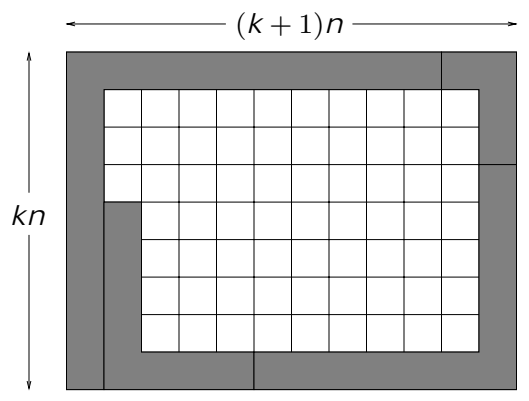

Figure 3: $N(k n,(k+1) n)$ with the first four removed strips marked, as implied by the parity theorem.

The process of removing each strip can be summarized in the following table describing how many squares are removed from the sides of the region, starting with $N(k n,(k+1) n)$, after each application of the parity theorem.

\begin{tabular}{c|l|l|l|l} 
App. & Left & Top & Right & Bottom \\
\hline 1 & $k n$ & $k n+1$ & - & - \\
2 & - & $n-1$ & $n$ & - \\
3 & - & - & $(k-1) n$ & $(k-1) n+1$ \\
4 & $2 n-1$ & - & - & $2 n-2$ \\
$\vdots$ & $\vdots$ & $\vdots$ & $\vdots$ & $\vdots$ \\
$4 j-3$ & $(k-2 j+2) n$ & $(k-2 j+2) n+1$ & - & - \\
$4 j-2$ & - & $(2 j-1)(n-1)$ & $(2 j-1)(n-1)+1$ & - \\
$4 j-1$ & - & - & $(k-2 j+1) n$ & $(k-2 j+1) n+1$ \\
$4 j$ & $2 j(n-1)+1$ & - & - & $2 j(n-1)$
\end{tabular}

Continue to apply the parity theorem until the $(\{i, i+1\} ; 1)$-strip removed is from an $(\{i, i+1\} ; 1)$-corner. To determine when this might happen, we need to solve any of the following equations, where the term subtracted from the left side of each refers to the squares occupied by previously removed corners.

$$
\begin{gathered}
k n-(2 j-1)=2 j(n-1)+1 \\
(k+1) n-(2 j-2)=(k-2 j+2) n+1
\end{gathered}
$$




$$
\begin{gathered}
k n-(2 j-2)=(2 j-1)(n-1)+1 \\
(k+1) n-(2 j-1)=(k-2 j+1) n+1
\end{gathered}
$$

These equations correspond to the final $(\{i, i+1\} ; 1)$-strip being removed from the lower left corner, the upper left corner, the upper right corner, and the lower right corner, respectively. The solution to (6) is $n=0$ or $k=2 j$, the solution to (7) is $n=1$ or $2 j-1=0$, the solution to (8) is $n=0$ or $k=2 j-1$, and the solution to (9) is $n=1$ or $2 j=0$. Since $n, k$, and $j$ are positive integers, the applications of the parity theorem cease in the manner described above when $n=1$ or when $2 j=k$ or $2 j-1=k$, depending on the parity of $k$.

Suppose that $n>1$, and let $k$ be even, setting $j$ to $k / 2$. After removing the $(2 k)^{\text {th }}$ strip, we are left with the subregion of $N(k n,(k+1) n)$ formed by removing the top $j$ rows, the bottom $j$ rows, the left $j+1$ columns, and the right $j$ columns. This is $N(k(n-1),(k+1)(n-1))$, so

$$
\#_{2} N(k n,(k+1) n)=\#_{2} N(k(n-1),(k+1)(n-1)) .
$$

If, on the other hand, $k$ is odd, let $j=(k+1) / 2$. After removing the $(2 k)^{\text {th }}$ strip from $N(k n,(k+1) n)$, the resulting subregion is $N(k n,(k+1) n)$ with the top $j$ rows, the bottom $j-1$ rows, the left $j$ columns, and the right $j$ columns removed. Since $k=2 j-1$, this once again gives (10).

Therefore, $\#_{2} N(k n,(k+1) n)=\#_{2} N(k, k+1)$ for all positive integers $k$ and $n$. Applying the parity theorem once to any corner of $N(k, k+1)$ indicates that

$$
\#_{2} N(k, k+1)=\#_{2} N(k-1, k)
$$

for all $k>1$. Therefore $\#_{2} N(k n,(k+1) n)=\#_{2} N(1,2)=1$ for all positive integers $k$ and $n$.

As mentioned previously, there are other ways to obtain this result, for example using Kasteleyn's formula. However, these methods tend to be much more analytic, and thus somewhat less intuitively clear, than the combinatorial proof presented here.

Corollary 4 studied $(\{s, t\} ; 1)$-corners, and we conclude this section by considering more general types of corners. Let $T(i, j, p)$ be the region with $i+p-1$ rows, whose rows from top to bottom consist of the following number of squares: $j, j+2, \ldots, j+2(p-1)$, $\ldots, j+2(p-1)$, where there are $i$ rows of $j+2(p-1)$ boxes. Similarly, let $D(i, j, p)$ be the region with $i+2(p-1)$ rows, whose rows from top to bottom consist of the following number of squares: $j, j+2, \ldots, j+2(p-1), \ldots, j+2(p-1), j+2(p-1)-2, \ldots$, $j+2, j$, where there are $i$ rows of $j+2(p-1)$ boxes. In each of these regions, the centers of the rows are aligned vertically. For $T(i, j, p)$ to have an even number of squares (and hence be possibly tilable), either $j$ or $i+p-1$ must be even. Similarly, for $D(i, j, p)$ to have an even number of squares, either $j$ or $i$ must be even.

The parity theorem applies to $T(i, j, p)$ and $D(i, j, p)$, and examples of such applications are given below. 
(a)

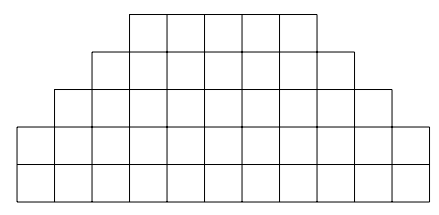

(b)

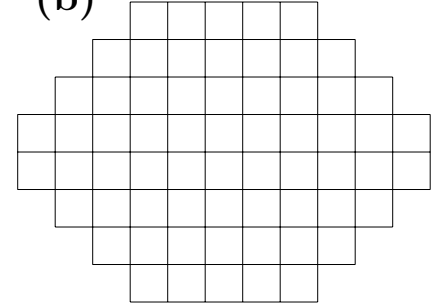

Figure 4: (a) The region $T(2,5,4)$. (b) The region $D(2,5,4)$.

Corollary 5. (a) $\#_{2} T(k, k, p)=0$.

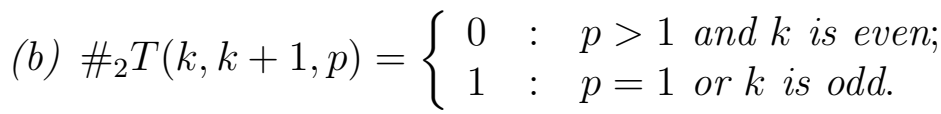

(c) $\#_{2} T(k, k+2, p)=\left\{\begin{array}{lll}0 & : & k \text { is odd } \\ 1 & : & k \text { is even. }\end{array}\right.$

(d) $\#_{2} T(k, 2 k-1, p)=0$.

(e) $\#_{2} T(k, 2 k, p)=1$.

(f) $\#_{2} T(k, 2 k+1, p)=0$.

(g) $\#_{2} T(k, 2 k+2, p)=1$.

Proof. Each of these follows inductively from (sometimes several) straightforward applications of the parity theorem.

(a) This follows immediately from Corollary 3.

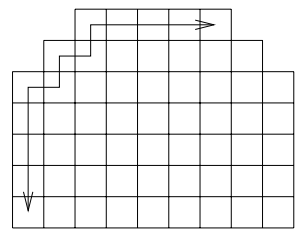

(b) $T(k, k+1,1)$ is a $k \times(k+1)$ rectangle, so the result for $p=1$ follows from Corollary 4 . For $p>1$, notice that $\#_{2} T(k, k+1, p)=\#_{2} T(k-1, k+1, p-1)$, so for $k>1$ we must use the answer from the subsequent part. $T(1,2, p)$ has one tiling.

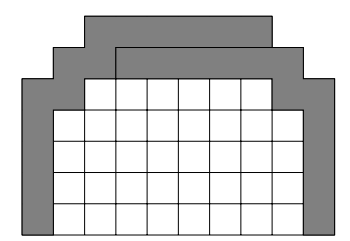


(c) Apply the parity theorem twice to see that $\#_{2} T(k, k+2, p)=\#_{2} T(k-2, k, p)$, and $\#_{2} T(1,3, p)=0$ for all $p$, while $\#_{2} T(2,4, p)=\#_{2} T(1,2, p)=1$ for all $p$.

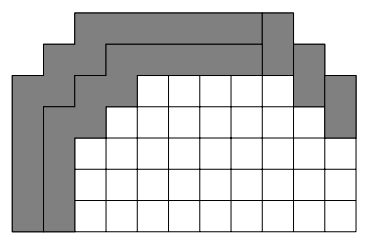

(d) Several applications of the parity theorem imply that $\#_{2} T(k, 2 k-1, p)=\#_{2} T(k-$ $2,2(k-2)-1, p)$, and this is 0 for both $k=1$ and $k=2$.

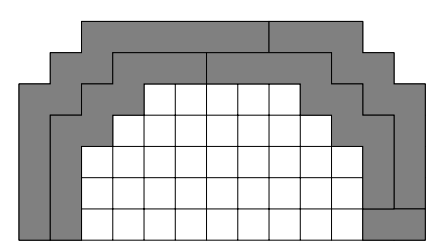

(e) $\#_{2} T(k, 2 k, p)=\#_{2} T(k-1,2(k-1), p)$ and $\#_{2} T(1,2, p)=1$.

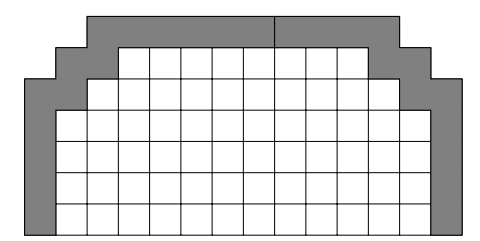

(f) After applying the parity theorem once, apply Corollary 3.

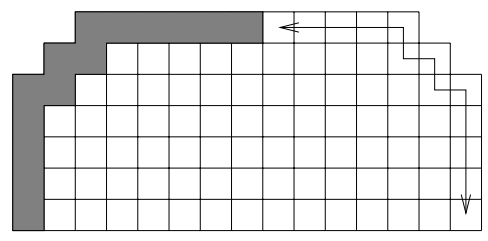

(g) Two applications of the parity theorem imply that $\#_{2} T(k, 2 k+2, p)=\#_{2} T(k-$ $1,2 k, p)$, and $\#_{2} T(1,4, p)=1$ for all $p$.

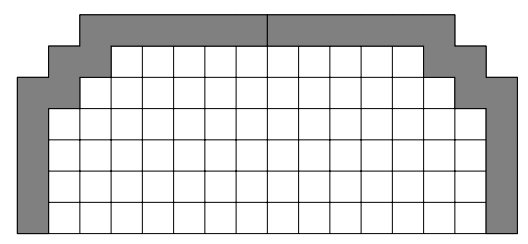

Corollary 6. (a) $\#_{2} D(k, k, p)=0$. 
(b) $\#_{2} D(k, k+1, p)=1$.

(c) $\#_{2} D(k, k+2, p)=0$.

(d) $\#_{2} D(k, 2 k-1, p)=\left\{\begin{array}{lll}0 & : & k \text { is odd } \\ 1 & : & k \text { is even. }\end{array}\right.$

(e) $\#_{2} D(k, 2 k+1, p)=0$.

Proof. As before, these results follow inductively from the parity theorem.

(a) This follows immediately from Corollary 3.

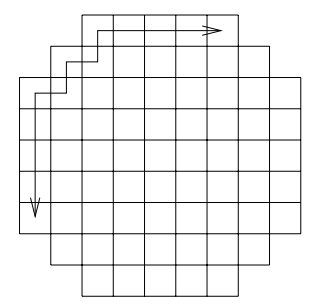

(b) Applying the parity theorem twice yields $\#_{2} D(k, k+1, p)=\#_{2} D(k, k+1, p-1)$, and $\#_{2} D(k, k+1,1)=1$.

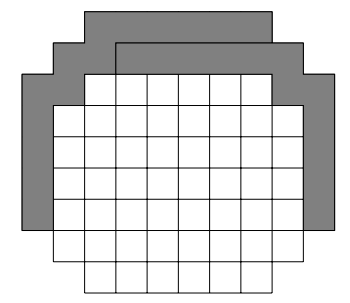

(c) If $p>1$, then this follows from Corollary 3 after one application of the parity theorem. If $p=1$, then $\#_{2} D(k, k+2,1)=\#_{2} D(k-2, k, 1)$, and this is 0 for $k=1$ and $k=2$.

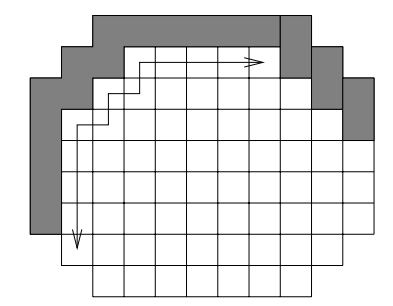

(d) $\#_{2} D(k, 2 k-1, p)=\#_{2} D(k-2,2(k-2)-1, p)$, and $\#_{2} D(1,1, p)=0$ for all $p$, while $\#_{2} D(2,3, p)=1$ for all $p$.

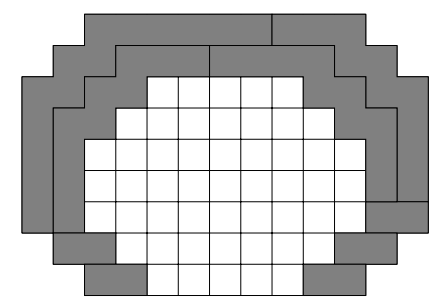


(e) After one application of the parity theorem, use Corollary 3.

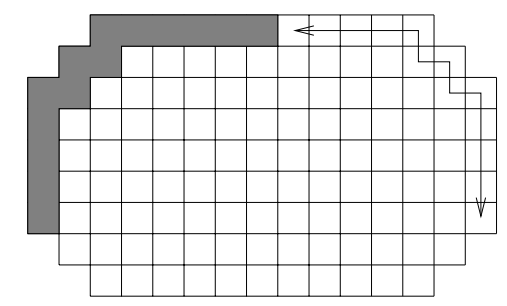

\section{References}

[1] M. Ciucu, Enumeration of perfect matchings in graphs with reflective symmetry, J. Combin. Theory, Ser. A 77 (1997) 67-97.

[2] P. W. Kasteleyn, The statistics of dimers on a lattice, I. The number of dimer arrangements on a quadratic lattice, Physica 27 (1961), 1209-1225.

[3] L. Pachter, Combinatorial approaches and conjectures for 2-divisibility problems concerning domino tilings of polyominoes, Electron. J. Comb. 4 (1997) R29.

[4] J. Propp, Enumeration of Matchings: Problems and Progress, in New Perspectives in Geometric Combinatorics, L. Billera, A. Björner, C. Greene, R. Simion, and R. P. Stanley, eds., MSRI Publications, vol. 38, Cambridge University Press, Cambridge, 1999, pp. 255-291.

[5] R. Tauraso, A new domino tiling sequence, Journal of Integer Sequences 7 (2004) 04.2.3. 